\title{
Generalization of wavelength matching to novel stimulus combinations*
}

\author{
KAY MALOTT, JAMES T. NORTHROP \\ and \\ ROBERT W. GRIFFEN \\ Western Michigan University, Kalamaźoo, Mich. 49001
}

\begin{abstract}
Pigeons' keypeck responses were reinforced in the presence of a bisected response key when both halves of the key were of equal wavelength (matching stimulus). Responses were extinguished when the halves were of different wavelength (nonmatching stimulus). Subsequent generalization testing, in which the wavelengths on the two halves of the key were varied among four values in the range from 501.3 to $605.0 \mathrm{~nm}$, gave evidence for generalization of the matching discrimination to novel wavelength combinations. Evidence against the possible contribution of intensity matching was cited. Failure of complete generalization was interpreted in terms of extinction gradients and degree of discrimination prior to testing.
\end{abstract}

Malott \& Malott (1970) and Malott, Malott, Svinicki, Kladder, \& Ponicki (1971) reported a series of studies concerning generalization of a single-response wavelength-matching discrimination. The circular response key was divided in half by a vertical black line. Pigeons' keypecking responses were reinforced when the two halves of the key were of equal wavelength (matching stimulus); in several of the studies, responses were extinguished when the halves differed in wavelength (nonmatching stimulus). Subsequent generalization tests involved the presentation of novel wavelengths or novel wavelength combinations. Evidence for generalization of matching (conceptual matching behavior) was obtained after very minimal training conditions. This was demonstrated by higher response rates in the presence of matching stimuli than in the presence of nonmatching stimuli.

Although these studies demonstrated that the single-key technique may be useful for the study of conceptual matching behavior, they may be criticized on the grounds that only two wavelengths were used in any one generalization test. A more stringent test for generalization of matching would involve presentations of a greater number of wavelengths and a greater portion of the visible continuum than was covered in the earlier experiments. Such an approach would be more likely to yield information regarding the relation of simple generalization and discrimination processes to the more complex process of stimulus matching. The present study is directed at this problem. This study also

*This work was supported by National Institute of Mental Health Grant 13178 to Western Michigan University, with Richard Malott as principal investigator. Richard Malott sponsors this paper and takes full editorial responsibility for it. attempts to eliminate the possible contribution of intensity matching discriminations which may have been present in the former studies.

\section{METHOD}

The Ss were 12 experimentally naive White-King barren hen pigeons. Purina pigeon grain served as the reinforcer and for additional feedings to maintain the Ss at $70 \%$ of their ad lib weights.

Two Lehigh Valley Electronics pigeon test chambers (No. 1519c) were used with the houselights off. Only one response key in each chamber was operative; it consisted of a transparent paddle behind a circular hole, $2.5 \mathrm{~cm}$ in diam. Visual stimuli were projected through the paddle from the rear by an Industrial Electronics Engineers, Inc. one-plane readout (Series 10) and GE 47 lamps. The circular stimulus was divided in half by a black vertical line, $0.6 \mathrm{~cm}$ wide. Intensity and wavelength were varied independently on the two halves. Kodak Wratten filters, Nos. $65,74,73$, and $72 \mathrm{~b}$, were used to obtain the four dominant wavelengths of 501.3 (blue), 538.0 (green), 576.0 (yellow), and $605.0 \mathrm{~nm}$ (red), respectively. A Kodak No. 96 neutral density 0.20 filter was used to vary intensity. Human observers judged that when this filter was placed over the brightest stimulus (green), it appeared to be slightly dimmer than the dimmest stimulus (red). Programming and recording of stimulus and response events were accomplished automatically through the use of solid-state digital switching circuitry and Sodeco electromechanical counters.

The Ss were assigned randomly to four groups of three Ss each. All groups received the same four $S+s$ (stimuli associated with reinforcement). The four $S+s$ were matching stimuli: blue (B-B), green (G-G), yellow (Y-Y), or red (R-R) on both halves of the key. Each group also received two $S-s$ (stimuli associated with extinction), but the groups differed in the wavelengths contained in the $S-s$. The $S-S$ were nonmatching stimuli: Group BG received S-s composed of blue on the left half of the key with green on the right (B-G) and green on the left half of the key with blue on the right (G-B); Group BY received B-Y and $Y-B$, Group BR received $B-R$ and $R-B$, and Group GY received G-Y and $Y-G$.

Under standard operant conditioning procedures, all Ss were trained to peck the response key in the presence of the four successively presented $\mathbf{S}+\mathbf{s}$. Responding was stabilized on a random interval 32-sec schedule of reinforcement (Farmer, 1963). The $S-s$ were then introduced. Stimuli were presented for 2-min intervals. The order of presentation of the six stimuli was random. Daily discrimination training sessions lasted for $50 \mathrm{~min}$.

Throughout training, the neutral density filter was placed over the right half of the stimulus, the left half, both halves, or neither half. These conditions were constant within sessions but varied between sessions. This was done in order to prevent intensity matching discriminations. Note, for example, that when the two halves of the key matched in wavelength, they might or might not match in intensity; reinforcement was thus received in the presence of both matching and nonmatching intensities.

When approximately equal response rates in the matching stimuli (stimuli matching in wavelength) were displayed and when the discrimination ratio, (response rate in the $\mathrm{S}+\mathrm{s}) /($ response rate in the $\mathrm{S}-\mathrm{s}$ ), was less than or equal to 0.3 for three consecutive training sessions, the first generalization test 


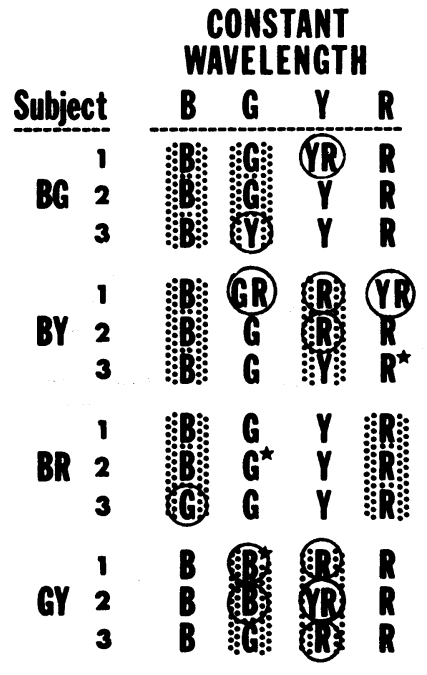

Fig. 1. Variable wavelength at which the peak of the generalization gradient occurred for each test. Two wavelengths are shown for a single test when equal response rates occurred in those stimuli. See text for further explanation.

was administered. Subsequent tests were administered after the same criteria were again met during continued training.

Wavelength generalization testing was conducted with no neutral density filter present. No novel wavelengths were presented; however, some of the combinations of wavelengths were novel. For each test, one wavelength, the "constant" wavelength, was always present on one or the other half of the key throughout the test; while the wavelength on the other half, the "variable" wavelength, was varied among the four available values in a random but repeated order. Each test was $28 \mathrm{~min}$ long and consisted of $56 \quad 30-\mathrm{sec}$ stimulus presentations, 7 presentations of each of the eight possible stimulus combinations in a predetermined random order. Because it was frequently found that the generalization gradients were relatively flat at the beginning of testing, the data to be presented were taken from only the last $16 \mathrm{~min}$ of testing. The constant wavelength was yellow in the first test, blue in the second, green in the third, and red in the fourth.

\section{RESULTS AND DISCUSSION}

Figure 1 presents the variable wavelength at which the peak of the generalization gradient occurred for each test. For example, the data for S2 in the BY group are shown in the fifth row; this S's gradient for the blue-constant test has a peak at blue, for the green-constant test the peak is at green, for the yellow-constant test it is at red, and for the red-constant test at red. A star indicates that the gradient was obtained from the entire test instead of from the last $16 \mathrm{~min}$; this was done whenever the number of responses at the maximum of the gradient was less than 40 during the last $16 \mathrm{~min}$, which was considered indicative of an unreliable gradient. In the three cases where this occurred, the numbers of responses at maximum during the last 16 min of testing were 0,4 , and 33 . All gradients used contained at least 40 responses at maximum.

Thirty-six of the 48 gradients have peaks at the variable wavelength which matches the constant wavelength, indicating matching behavior. The 12 that do not are circled in Fig. 1. Nine of the 12 that do not have peaks at matching values were obtained when a training S- wavelength was held constant in the test; shaded areas indicate constant wavelengths which are the same as the training $\mathrm{S}$ - wavelengths. The remaining three were obtained in cases where maximal responding occurred at more than one value, indicating a lack of discrimination among wavelengths.

In summary, when a good discrimination between wavelengths exists (i.e., when sharp gradients occur), matching is consistently displayed except in some cases where $\mathrm{S}-$ training wavelengths are held constant in the tests. These failures to demonstrate matching when an $S$ - training wavelength is held constant may be related to the extinction gradients studied by Honig (1961). With extinction gradients over wavelength, one would expect a minimum at an $\mathrm{S}$ - wavelength, with response rate increasing as a function of the distance from the $S-$ wavelength. Two of the gradients from the yellow-constant test for the BY group, for example, fit this description; these gradients have minima at blue and maxima at red. The rest of the gradients under discussion may be similarly interpreted. Particularly significant is the fact that five of the six gradients obtained with an $\mathrm{S}$ - training color held constant in the GY group have peaks as predicted by an extinction gradient analysis. (An interpretation in terms of the peak-shift phenomenon, as studied by Hanson (1959) or Guttman (1965), is probably not relevant to these data, since the test stimuli are spaced too far apart to detect such shifts.)

On the other hand, some of the data seem to indicate generalization of the matching discrimination. Four of the Ss demonstrate matching for all constant wavelengths. Two of these are in the BR group ( $\mathrm{S} 1$ and $\mathrm{S} 2$ ), one is in the BY group (S3), and one is in the BG group (S2). These gradients do not all exhibit minima at a wavelength used in training $S-S$ and maxima at a wavelength distant from that $S$ - wavelength, as would be the case with extinction gradients. Other gradients also contradict an extinction gradient interpretation. For example, although $\mathrm{S} 2$ in the BY group shows a peak at red for both the yellow-constant and red-constant tests (which is consistent with an extinction gradient interpretation), the green-constant gradient shows matching.

An interpretation of generalized wavelength matching in terms of the summation of simple wavelength generalization gradients has been presented earlier (Malott \& Malott, 1970; Malott et al, 1971). Although such an interpretation can never be totally ruled out using the wavelength continuum, Malott et al indicate that such an interpretation is probably unlikely. Unless such an interpretation is, in fact, applicable to the present experiment, it seems clear that conceptual matching behavior has been demonstrated.

A fact which may be of significance is that three of the four Ss that demonstrate matching in the presence of 
all four constant wavelengths had at least 10 consecutive sessions prior to Test 1 in which the discrimination ratio was less than or equal to 0.3; all remaining Ss had only three or four such sessions. This may indicate that a greater consistency or "degree" of discrimination in training leads to improved conceptual matching behavior.

Pilot experiments from this laboratory indicate that Ss trained as in the present experiment will show no intensity matching; tests were conducted in which intensity was varied in a manner similar to that in which wavelength was varied in the current experiment, with no indication of an intensity matching discrimination.

\section{REFERENCES}

Farmer, J. Properties of behavior under random interval reinforcement schedules. Journal of the Experimental Analysis of Behavior, 1963, 6, 607-612.

Guttman, N. Effects of discrimination formation on generalization. In D. I. Mostofsky (Ed.), Stimulus generalization. Stanford: Stanford University Press, 1965

Hanson, N. M. Effects of discrimination training on stimulus generalization. Journal of Experimental Psychology, 1959, 58 , 321-334.

Honig, W. K. Generalization of extinction on the spectral continuum. Psychological Record, 1961, 11, 269-278.

Malott, R. W., \& Malott, M. K. Perception and stimulus generalization. In W. C. Stebbins (Ed.), Animal psychophysics: The design and conduct of sensory experiments. New York: Appleton-Century-Crofts, 1970.

Malott, R. W., Malott, M. K., Svinicki, J. G., Kladder, F., \& Ponicki, E. An analysis of matching and non-matching behavior using a single-key free-operant procedure. Psychological Record, 1971, 21, 545-564.

(Received for publication October 31, 1972.) 